\title{
Treatment outcomes of midfacial segment pain: experience from the Liverpool multi-disciplinary team facial pain clinic*
}

\author{
Samuel C. Leong', Lepa Lazarova', Hoo K. Tsang², Gyorgy Banhegyi \\ Department of Otolaryngology - Head and Neck Surgery Royal Liverpool University Hospital, Liverpool, United Kingdom \\ 2Department of Anesthesiology and Pain Management, Royal Liverpool University Hospital, Liverpool, United Kingdom
}

\author{
Rhinology 53: 35-40, 2015 \\ DOl:10.4193/Rhino14.156 \\ *Received for publication: \\ July 4, 2014 \\ Accepted: September 4, 2014
}

\begin{abstract}
Objectives: Midfacial segment pain (MSP) has the characteristics of tension-type headache which is confined to the midface corresponding to the second division of the trigeminal nerve. This review presents treatment outcomes of MSP patients managed at the Multi-disciplinary Team (MDT) Facial Pain Clinic in Liverpool.
\end{abstract}

Methodology: Prospective clinical outcome performed in a tertiary referral centre for complex facial pain syndromes.

Main outcome measures: Sino-Nasal Outcome Test (SNOT). Clinical "success" was defined as an improvement in total SNOT score of $>9$ points and a reduction of the ear-facial symptoms sub-domain score by $\geq 50 \%$ from baseline.

Results: The average age of the cohort was 49 years, with an average follow-up of 12 months. The overall pre-treatment total SNOT-22 score was 59.5 which improved significantly to 42 at latest follow-up. Although the average scores of all sub-domains improved, only the ear-facial symptoms and psychological issues sub-domains achieved statistical significance. When the criterion for success was applied, nine patients fulfilled this definition at an average of 12 months follow-up. The baseline total SNOT score in this cohort improved from 60.6 to 19.7. Half of these patients achieved success within 18 months of commencing treatment and the probability of attaining success at long-term follow-up was high.

Conclusions: Treatment of midfacial segment facial pain is complex and requires follow-up to achieve any meaningful clinical outcome.

Key words: tension headache, sinus, facial, pain, migraine

\section{Introduction}

Midfacial segment pain (MSP) has the characteristics of tensiontype headache, with the exception that it affects the midface confined to the second division of the trigeminal nerve ${ }^{(1)}$. Patients typically describe a feeling of pressure, heaviness or tightness and in the absence of nasal airway obstruction they may say that their nose feels blocked. The symptoms are symmetrical and may involve the nasion, the bridge of the nose, either side of the nose, the peri-orbital region, retro-orbitally or across the cheeks. This is commonly mistaken as being "sinus headaches" or "rhinogenic headache", and has resulted in patients being referred with assumed sinusitis ${ }^{(2)}$. However, normal endoscopy and computed tomography of the paranasal sinuses are typical in these patients ${ }^{(3)}$.

The Sino-Nasal Outcome Test is a validated and widely adopted patient-reported outcome measure for a variety of sino-nasal procedures such as sinus surgery for chronic rhinosinusitis, septal surgery ${ }^{(4)}$, rhinoplasty ${ }^{(5)}$ and obstructive sleep apnoea ${ }^{(6)}$. Browne et al. ${ }^{(7)}$ described four distinct subscales within the 
SNOT. The first covers rhinologic symptoms and contains five questions: need to blow nose, sneezing, runny nose, postnasal discharge, and thick nasal discharge. The second covers ear and facial symptoms and contains four questions: ear fullness, dizziness, ear pain, and facial pain/pressure. The third covers sleep function and contains three questions: difficulty falling asleep, waking up at night, and lack of a good night's sleep. The fourth covers psychological issues and contains six questions: fatigue, reduced productivity, reduced concentration, frustration/restlessness/irritability, sadness, and embarrassment. This clinical group had previously described the utility of the Sino-Nasal Outcome Test-22 (SNOT-22) in MSP patients and demonstrated that the elevated overall scores were comparable to patients suffering with chronic rhinosinusitis (CRS) ${ }^{(8)}$. In addition, the earfacial symptom, sleep function and psychological subdomains within SNOT-22 were higher in MSP patients than that observed in CRS patients. It was proposed that the SNOT-22 could be a suitable tool to evaluate treatment outcomes in ORL clinics.

\section{Materials and methods}

\section{Ethical considerations}

This retrospective clinical outcomes study was approved by the Department of Clinical Audit and Information (reference 394510/11).

\section{Patients}

Patients with midfacial segment pain (Table 1) were identified from the MDT database. The review period was limited to between June 2011 and June 2013. This clinic was established in June 2011 and provides a tertiary service for patients with complex facial pain syndromes in Liverpool. These patients were usually referred from pain management and general ORL clinics after being assessed for sinonasal pathology, and were endoscopy-negative and computer tomography (CT)-negative at the time. Patients were re-assessed against the International Headache Society classification for headache disorders, cranial neuralgia and facial pain. Nasendoscopy was repeated to confirm the presence or absence of sinonasal pathology. All patients routinely completed the SNOT-22 questionnaire prior to their consultation. In this study, the SNOT scores prior to commencement of medical treatment for facial pain was evaluated and regarded as baseline. Treatment and follow-up protocols adhere to recommendations by the National Institute for Health and Clinical Excellence (NICE) for the management of neuropathic pain (Figure 1).

No previous studies have utilised the SNOT instrument to evaluate treatment outcomes of facial pain. A recent study by Agius et al. ${ }^{(9)}$ defined treatment success as more than $50 \%$ reduction of pain frequency or intensity or both, based on a 10-point visual analogue scale. Hopkins et al. ${ }^{(10)}$ reported that the smallest change in SNOT-22 score that can be detected by a patient was 8.9 points, but this was based on patients with chronic rhinosinusitis. For the purpose of this study, "success" was defined as an improvement in total SNOT score of $>9$ points and a reduction of the ear-face symptoms sub-domain score by $\geq 50 \%$ from baseline. Follow-up was defined as the period when "success" was achieved, or the prevailing SNOT at latest clinic follow-up. Patients were excluded if they had not yet been re-assessed, failed to attend follow-up, or lost to follow-up.

\section{Statistics}

Statistical analysis was performed using the SigmaPlot software package version 12 (Systat Software, Inc., CA, USA). Student's t

Table 1. Diagnostic criteria for midfacial segment pain (1).

Midfacial segment pain

1. A symmetric sensation of pressure or tightness. Some patients might say that their nose feels blocked, although they have no nasal airway obstruction.

2. Involves the areas of the nasion, under the bridge of the nose, either side of the nose, the peri- or retro-orbital regions, or across the cheeks. The symptoms of tension-type headache often coexist.

3. There might be hyperesthesia of the skin and soft tissues over the affected area.

4. Normal nasal endoscopy.

5. Normal CT of the paranasal sinuses.

6. The symptoms can be intermittent ( $<15$ days/month) or chronic ( $>15$ days/month).

7. No consistent exacerbating or relieving factors.

8. No significant nasal symptoms (note that approximately $20 \%$ of most populations have intermittent or persistent allergic rhinitis that may occur incidentally in this condition). 


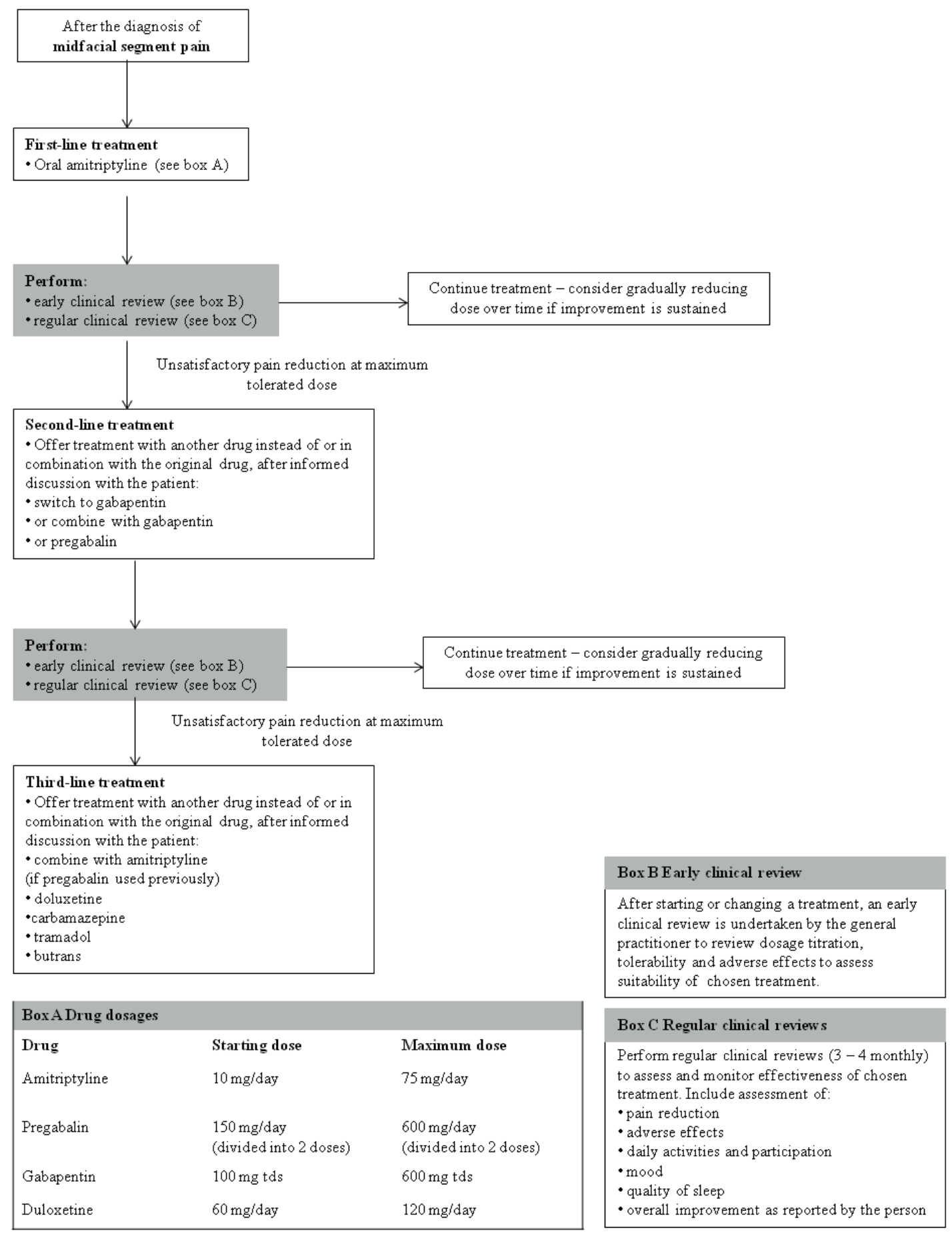

Figure 1. Flow-chart illustrating the management algorithm used by the Liverpool Multi-Disciplinary Facial Pain team.

test was used to compare the SNOT scores or when normality test (Shapiro-Wilk) failed, the Mann-Whitney $U$ test was utilised instead. A p-value $<0.05$ was considered statistically significant.

\section{Results}

Twenty patients ( 18 females) fulfilled the inclusion criteria for this review. The average age of this cohort was 49 years (median 50 , range $25-70$ ), with an average follow-up of 12 months (median 11 months, range 3 - 33). The overall pre-treatment total SNOT-22 score was 59.5 (standard deviation (SD) 20.7) which improved significantly ( $p<0.05$ ) to 42 (SD 26.6) at latest follow-up (Table 2). Although the average scores of all subdomains improved, only the ear-facial symptoms and psychological issues sub-domains achieved statistical significance (Figure 2). When individual patient data were analysed, 13 (65\%) had improved total SNOT scores with an average improvement of 32 


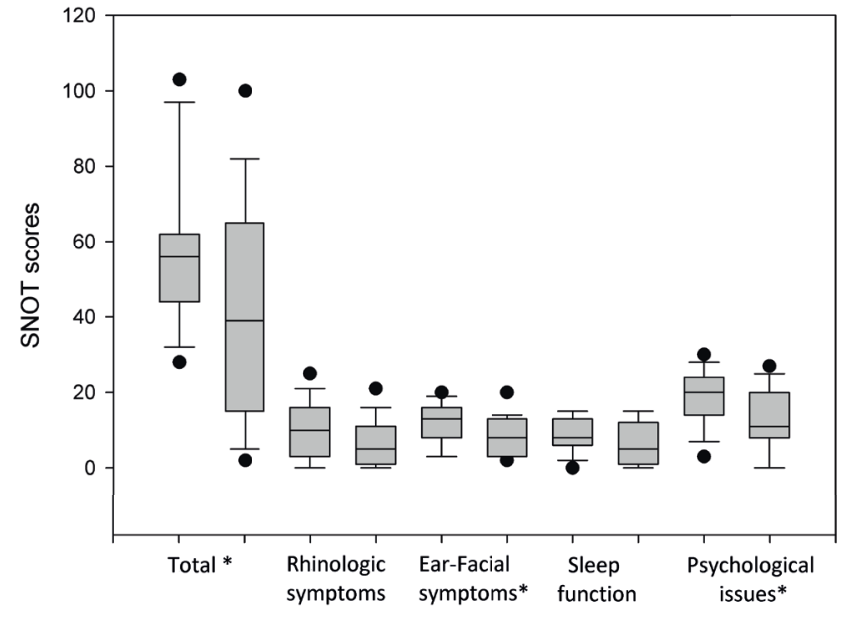

Figure 2. Box-plots comparing baseline and follow-up total and sub-domain SNOT scores; ${ }^{*}=p<0.05$.

points (range 3 - 79) whilst six (32\%) reported worsening total SNOT (average 11.2, range 3 - 21) and one remained unchanged. The baseline SNOT scores of those who reported improved and worsened scores were not significantly dissimilar, suggesting a degree of homogeneity in the disease impact between the subgroups.

When the criterion for success was applied, nine (45\%) patients fulfilled this definition at an average of 12 months (SD 7.5) follow-up. The baseline total SNOT score in this cohort improved from 60.6 (SD 19.9) to 19.7 (SD 13.4). In contrast, the average

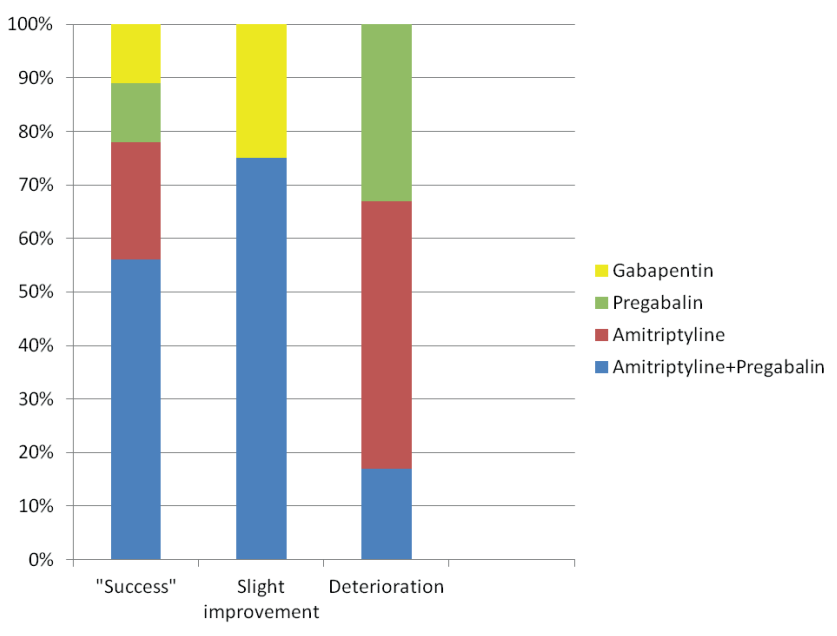

Figure 4. Stacked box-plot showing the distribution of treatment combinations between MSP patients who achieved success, those who had slight improvement in reported SNOT and those who had deteriorated SNOT scores.

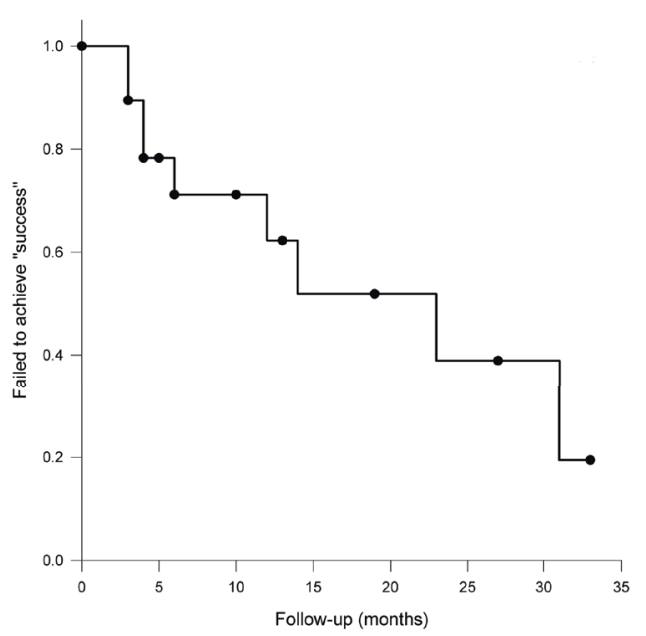

Figure 3. Kaplan-Meier curve illustrating probability of not achieving successful response to treatment.

baseline pre-treatment score for those who did not achieve success had deteriorated from 58.5 (SD 22.2) to 60.3 (19.6), although this difference was not statistically significant. No statistically significant difference was observed in the baseline scores between the subgroups. Nevertheless, Kaplan-Meier analysis showed that $50 \%$ achieved success within 18 months of commencing treatment and the probability of attaining success at long-term follow-up was high (Figure 3). Over half of patients who achieved success were on amitriptyline and pregabalin dual-therapy (Figure 4). The majority of patients who had improved SNOT but did not fulfil the criteria for "success" were on combination amitriptyline and pregabalin.

\section{Discussion}

We believe the results presented here have provided a realistic impression of the challenges faced when managing patients with MSP. This may be invaluable to either clinicians delivering a comprehensive rhinology service or those in more general ORL practice. In addition, it provides comparative data for audit undertaken by other facial pain services. In our experience, positive response to treatment was not universally observed in all patients. In fact, only $69 \%$ (9 of 13) of those who had improved SNOT scores had a significant improvement, defined as success in this study. Although this criterion was an arbitrary definition, no other patients had comparable overall or subdomain specific SNOT score improvement which suggests that the criteria used in this study may be a robust indication of true clinical response. It must also be remembered that in order to attain meaningful clinical response, close long-term follow-up is required with flexibility to modify treatment combinations and dosages. In our study, $50 \%$ of those who achieved success did so within 18 months of commencing treatment; the most common combination being amitriptyline and pregabalin. 
Table 2. Summary of total and subdomain SNOT scores ( mean, standard deviation) before commencement of treatment and at latest follow-up. * significant difference $(p<0.05)$ compared to baseline score.

\begin{tabular}{|ccccc|}
\hline & $\begin{array}{c}\text { Pre-treat- } \\
\text { ment } \\
(\mathbf{n}=\mathbf{2 0})\end{array}$ & $\begin{array}{c}\text { At latest } \\
\text { follow-up } \\
(\mathbf{n}=\mathbf{2 0})\end{array}$ & $\begin{array}{c}\text { Achieved } \\
\text { "success" } \\
(\mathbf{n}=9)\end{array}$ & $\begin{array}{c}\text { Did not } \\
\text { achieve } \\
\text { "success" } \\
(\mathbf{n}=11)\end{array}$ \\
\hline Total & $59.5(20.7)$ & $42.0(26.6)$ & $19.7^{*}(13.4)$ & $60.3(19.6)$ \\
\hline $\begin{array}{c}\text { Rhinologic } \\
\text { symptoms }\end{array}$ & $10.1(7.1)$ & $7.1(6.0)$ & $4.1^{*}(4.5)$ & $9.5(6.1)$ \\
\hline $\begin{array}{c}\text { Ear and } \\
\text { facial } \\
\text { symptoms }\end{array}$ & $11.7(5.2)$ & $8.1^{*}(5.1)$ & $3.7^{*}(1.2)$ & $11.7(4.1)$ \\
\hline $\begin{array}{c}\text { Sleep func- } \\
\text { tion }\end{array}$ & $8.7(4.5)$ & $6.2(5.5)$ & $1.8^{*}(2.9)$ & $9.8(4.4)$ \\
\hline $\begin{array}{c}\text { Psychologi- } \\
\text { cal issues }\end{array}$ & $19.1(7.3)$ & $12.5^{*}(8.6)$ & $5.9^{*}(5.2)$ & $17.9(6.8)$ \\
\hline
\end{tabular}

Although the treatment protocol differed from that of Agius et al. (9), our results appear to corroborate with the observed variability of response to treatment. Nearly a quarter of the treatment cohort failed to meet their criteria for success. The discrepancies observed between our studies were due to different outcome measures used but more importantly, underscore the fact that treatment of neuropathic pain is complex, has variable clinical response and carries a significant psychological overlay. For example the impact of relaxation therapy, which is routinely offered to patients, was not evaluated in this report ${ }^{(10)}$. The heterogeneous treatment combination needed to achieve "success" shows that a meaningful clinical response does not necessarily follow conventional treatment algorithms, and that a degree of flexibility is needed ${ }^{(11)}$. The treatment protocol adopted by this MDT reflects the corporate experience of all clinicians involved. This is another significant deviation from the treatment regime reported by Agius et al ${ }^{(9)}$.
This was a retrospective point-in-time review of clinical outcomes from a small cohort of patients. Although it would have been ideal to have the entire cohort complete a set follow-up period to assess the level of response of each patient, the threshold period before maximal response can be recorded remains unknown in neuropathic pain. It can also be argued that the SNOT was an inappropriate tool to measure clinical outcomes in non-sinogenic facial pain. More disease specific questionnaires such as the Brief Pain Inventory-Facial Pain (BPI-FP) exist but unlikely to be familiar to the majority of ORL clinicians. We believe the SNOT was a suitable compromise which considered symptomatology, functional and psychological variables. Nevertheless, data from the BPI-FP is routinely collated in our clinic and how this correlates with the SNOT subdomain is being investigated ${ }^{(12)}$. Whilst we recognise that the visual analogue scale (VAS) is a commonly used instrument to assess clinical outcome, we decided against using a VAS as feedback from our patients and early analysis of available data suggested that the VAS as a simple metric did not correlate with the global changes of symptomatology and psychology over time.

\section{Conclusion}

Treatment of midfacial segment facial pain is complex and time-consuming. Definition of true "success" requires further validation which at present remains elusive. Needless to say, long-term follow-up of such patients is required to achieve any meaningful clinical outcome.

\section{Authorship contribution}

SCL: study concept, data collection and analysis, drafting of manuscript; LL: data collection; HKT: data collection; GB: data collection

Best poster prize at the North of England ENT society meeting. Liverpool 14 March 2014.

\section{Conflicts of interest}

No conflict of interest.

\section{References}

1. Jones NS. Midfacial segment pain: implications for rhinitis and rhinosinusitis. Clin Allergy Immunol. 2007; 19: 323-333.

2. Daudia AT, Jones NS. Facial migraine in a rhinological setting. Clin Otolaryngol Allied Sci. 2002; 27: 521-525.

3. Kamani T, Jones NS. 12 minute consultation: evidence based management of a patient with facial pain. Clin Otolaryngol. 2012; 37: 207-212.

4. Buckland JR, Thomas S, Harries PG. Can the Sino-nasal Outcome Test (SNOT-22) be used as a reliable outcome measure for successful septal surgery? Clin Otolaryngol Allied
Sci. 2003; 28: 43-47

5. Poirrier AL, Ahluwalia S, Goodson A, Ellis M, Bentley M, Andrews P. Is the Sino-Nasal Outcome Test-22 a suitable evaluation for septorhinoplasty? Laryngoscope. 2013; 123: 76-81.

6. Lachanas VA, Woodard TD, Antisdel JL, Kountakis SE. Sino-nasal outcome test tool assessment in patients with chronic rhinosinusitis and obstructive sleep apnea. ORL J Otorhinolaryngol Relat Spec. 2012; 74: 286-289.

7. Browne JP, Hopkins C, Slack R, Cano SJ. The Sino-Nasal Outcome Test (SNOT): can we make it more clinically meaningful?
Otolaryngol Head Neck Surg. 2007; 136: 736-741.

8. Leong SC, Tsang HK, Wilkie MD, Banhegyi G. Characterisation of patients with endoscopy-negative, computer tomographynegative midfacial segment pain using the sino-nasal outcome test. Rhinology. 2014; 52: 78-83.

9. Agius AM, Jones NS, Muscat R. A randomized controlled trial comparing the efficacy of low-dose amitriptyline, amitriptyline with pindolol and surrogate placebo in the treatment of chronic tension-type facial pain. Rhinology. 2013; 51: 143-153.

10. Hopkins C, Gillett S, Slack R, Lund VJ, 
Browne JP. Psychometric validity of the 22-item Sinonasal Outcome Test. Clin Otolaryngol. 2009; 34: 447-454

11. Chaparro LE, Wiffen PJ, Moore RA, Gilron I. Combination pharmacotherapy for the treatment of neuropathic pain in adults. Cochrane Database Syst Rev. 2012 Jul 11;7:CD008943. doi: 10.1002/14651858. CD008943.pub2.

12. Leong SC, Tsang HK, Banhegyi G. The Utility of the Sino-Nasal Outcome Test (SNOT) as a Treatment Outcome Measure in Nonsinogenic Facial Pain Syndrome. Ann Otol Rhinol Laryngol. Nov. 2014; [Epub ahead of print].
Mr. Samuel C. Leong, MPhil FRCSEd(ORL-HNS)

Division of Rhinology and Skull Base Surgery

Department of Otolaryngology Head and Neck Surgery

University Hospital Aintree Liverpool L9 7AL

United Kingdom

Email: Icheel@doctors.org.uk

\section{ADVERTISEMENT}

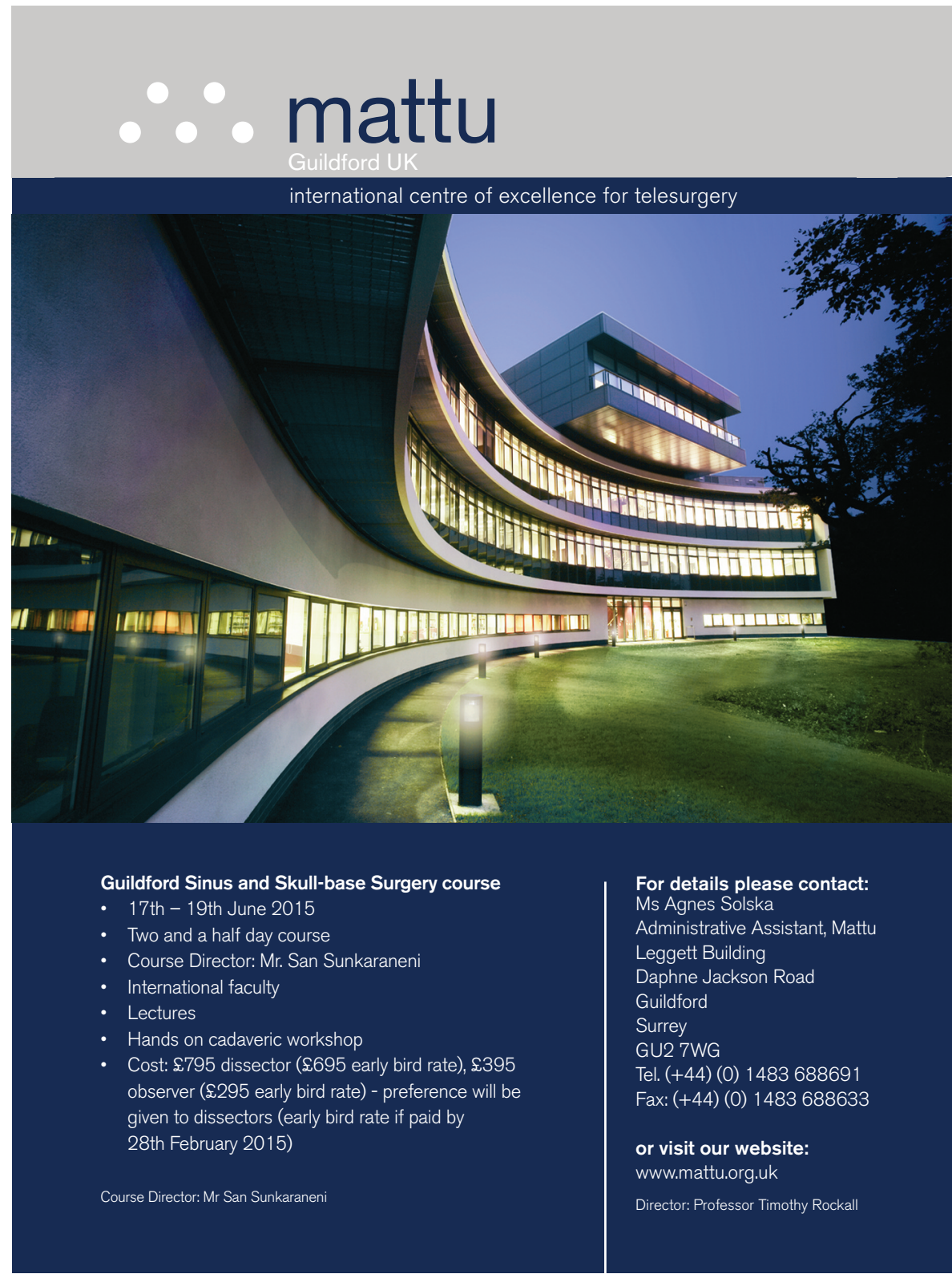

